Instituto Internacional de Investigación y Desarrollo Tecnológico Educativo INDTEC, C.A.

DOI: https://doi.org/10.29394/Scientific.issn.2542-2987.2018.3.8.6.121-137

OAl-PMH: http://www.indteca.com/ojs/index.php/Revista Scientific/oai

\title{
Metacognición: Razonamiento Hipotético y Resolución de Problemas
}

\author{
Autores: Jaime Iván Ullauri Ullauri \\ Universidad Nacional de Educación, UNAE \\ jaime.ullauri@unae.edu.ec \\ Azogues, Ecuador \\ Carol Ivone Ullauri Ullauri \\ Universidad Nacional de Educación, UNAE \\ carol.ullauri@unae.edu.ec \\ Azogues, Ecuador
}

\section{Resumen}

El presente trabajo contempla una reflexión teórica precisa y sucinta sobre el proceso metacognitivo de razonamiento hipotético como capacidad propia del desarrollo cognitivo de los niños durante la tercera infancia y como este incide en la resolución de problemas lógico-matemáticos, sobre la perspectiva cognitiva de Flavell y Sternberg en comunión con el proceso resolutorio de problemas de Polya. Esta investigación comprendió el estudio bibliográfico pertinente, teniendo como horizonte poner en evidencia los procesos de razonamiento hipotético en la resolución de problemas matemáticos, concluyendo con la importancia de la enseñanza y desarrollo de procesos cognitivos básicos en educación que fomenten el surgimiento de procesos metacognitivos, permitiendo al niño trabajar sobre su tarea, repensarla y en lo posible solucionarla. Desde esta perspectiva este trabajo constituye una panorámica de fácil acceso y comprensión para los profesionales noveles que se especializan en psicología y educación. problemas.

Palabras clave: cognición; pensamiento; razonamiento; resolución de 


\title{
Metacognition: Hypothetical Reasoning and Problem Solving
}

\begin{abstract}
The present work contemplates a precise and succinct theoretical reflection on the metacognitive process of hypothetical reasoning as a proper capacity of the cognitive development of children during the third childhood and how it affects the logical-mathematical problem solving, on the cognitive perspective of Flavell and Sternberg in communion with the resolving process of Polya's problems. This investigation comprised the pertinent bibliographical study, having like horizon to put in evidence the hypothetical reasoning processes in the resolution of mathematical problems, concluding with the importance of the teaching and development of basic cognitive processes in education that foment the sprouting of metacognitive processes, allowing the child will work on his task, rethink it and, as far as possible, solve it. From this perspective, this work constitutes an overview of easy access and understanding for new professionals who specialize in psychology and education.
\end{abstract}

Keywords: cognition; thinking; reasoning; problem solving. 


\section{Introducción}

El presente trabajo conduce a un recorrido de aproximación teórica sobre el desarrollo cognitivo y metacognitivo de los niños durante la tercera infancia (7-11 años de edad) en correspondencia con los fundamentos teóricos establecidos por Piaget (1991a) y Flavell (2000a). El fin último de este trabajo es poner en evidencia los aspectos teóricos más relevantes acerca del desarrollo del razonamiento lógico como un proceso metacognitivo en la resolución de problemas matemáticos en los niños.

Desde la teoría cognitiva de Flavell (2000b): se establecen las categorías conceptuales y etapas evolutivas del pensamiento, aquella transición cognitiva comprendida entre la segunda y tercera infancia, así desde esta perspectiva el autor considera que el conocimiento metacognitivo puede conducir al niño a asimilar estímulos que se encuentran en su contexto, asimilación que empata necesariamente con sus intereses, capacidades y metas. Sternberg (2011a): afirma que estas capacidades tienen que poner en manifiesto la compresión como la génesis del proceso que el niño desarrolla para solucionar un problema, esta compresión problemática es la que por medio de la evocación-memoria conduce a la activación de conocimientos previos, desde la cual se pueden producir diversas soluciones a un mismo problema.

\section{Teoría y conceptos}

\subsection{Cognición}

Es importante aclarar conceptos base que permitan construir una panorámica sobre el desarrollo cognitivo y sus implicaciones en el desarrollo integral del niño. Flavell (2000c): integra dentro del desarrollo cognitivo el desarrollo de procesos mentales superiores "correspondientes a entidades psicológicas como los saberes o conocimientos (knowledge), la conciencia, la inteligencia, el pensar, imaginar, crear, generar planes y estrategias, razonar, 
inferir, resolver problemas, conceptualizar, clasificar y relacionar, simbolizar y posiblemente fantasear y soñar." (Flavell, 2000d, pág. 13). Desde otra perspectiva Dorsch (2005): abarca la concepción de cognición y su desarrollo como un término genérico para denominar a todos los "procesos o estructuras que se relacionan con la consciencia y el conocimiento, como la percepción, el recuerdo (reconocimiento), la representación, el concepto, el pensamiento, y también la conjetura, la expectación, el plan” (pág. 121).

En conjunción con estas definiciones Ullauri, conceptualiza la cognición como:

La cognición no es tan solo un proceso o un conjunto de procesos que posibilitan al ser humano resolver sus problemas, la cognición es un conjunto de destrezas y competencias que permiten al ser humano ser 'capaz de', esta capacidad se traduce en poder esbozar y establecer relaciones lógicas, que solo las puede realizar el ser humano como tal (Ullauri, 2013a, pág. 18).

Así de esta manera "capaz de" se determina que el niño puede establecer de forma lógica estrategias para la resolución de problemas "se tiene que entender a la cognición no solo como una unidad mental de inteligencia sino como una unidad general del pensamiento, que permite al ser humano construir y reconstruir" (Ullauri, 2013b, pág. 18).

\subsection{Metacognición}

Para Bruner (1995): la metacognición es en primera instancia una habilidad, que permite al niño pensar sobre su pensamiento, posibilitándolo tener conciencia sobre la situación para poder resolverla, indicando además la clasificación por niveles de pensamiento: en el primer nivel se encuentran los procesos básicos de pensamiento que son innatos, en el segundo nivel encontramos la capacidad de recuerdo y estrategias adecuadas para estar 
alfabetizados culturalmente y en el tercer nivel los procesos y estrategias de pensamiento que son evocadas de manera consiente.

Desde la perspectiva de Flavell (2000e): considera a la metacognición como el cuarto nivel, el "nivel más alto" de la actividad mental del niño, que involucra los procesos más complejos del pensamiento, propios de la mente consciente. Para el autor en la tercera infancia los aspectos básicos en los que enmarca la metacognición son: a). las propias capacidades cognitivas, b). las tareas, c). las estrategias metacognitivas. Así mismo para Hacker (1998a): la metacognición integra: a). el conocimiento y b). la regulación metacognitiva, entendido el primero como aquel conocimiento que se posee sobre el propio conocimiento, que involucra también sus estados cognitivos y afectivos, interpretándolos y reinterpretándolos, en tanto que la regulación metacognitiva es el proceso por el cual el niño es capaz de controlar y regular los procesos cognitivos.

Mientras que desde la teoría de la mente Wellman (1985), postula etapas en las que se construye el conocimiento y la regulación metacognitiva son: i). existencia, ii). distinción de procesos, iii). integración, iv). conocimiento de las variables y v). monitoreo cognitivo.

\section{Desarrollo cognitivo en la segunda y tercera infancia}

La principal característica del pensamiento del niño en esta etapa del desarrollo es el avance a un pensamiento lógico, en el que va abandonando la intuición y el egocentrismo. Así desde esta mirada Piaget, incluye en esta etapa el estadio operacional concreto, espacio del desarrollo en la que los niños logran dominar las nociones de conservación, transitividad, inclusión de clase, la clasificación múltiple y la seriación, así como inicia la comprensión de la reversibilidad, la identidad y las nociones y comprensión de conceptos lógicos (Piaget, 1991b, pág. 54).

Flavell (2000f): en el estadio de operaciones formales concretas 
visibiliza los procesos cognitivos que se suceden durante la segunda y tercera infancia de la siguiente manera: en la segunda infancia se presenta procesos cognitivos como: i). apariencias percibidas, ii). centraciones, iii). estados y iv). irreversibilidad, mientras en correspondencia a cada uno de ellos en la tercera infancia se presentan ya desarrollados como: i). realidad inferida, ii). descentración, iii). transformaciones, iv). reversibilidad, determinando que la naturaleza del proceso de razonamiento hipotético es metacognitiva y que se presenta desde la tercera infancia.

Durante la segunda infancia (2-7 años), se evidencia un desarrollo en las competencias básicas de manera progresiva con presencia de la intuición y egocentrismo, en la tercera infancia (7-11 años), se potencializa las operaciones concretas y el pensamiento lógico, como afirma Flavell (2000g): "el desarrollo de estas que avanza desde la ausencia total de competencias a la presencia de las más avanzadas" (pág. 74). En torno a este aspecto Flavell indica además que la centración, las apariencias percibidas y la irreversibilidad son condiciones de la segunda infancia, mientras que la descentración, la realidad inferida y reversibilidad son condiciones de la tercera infancia.

\subsection{Metaconocimiento}

El desarrollo de procesos metacognitivos conlleva al individuo a trabajar en metaconocimientos, a los que Flavell (2000h): define como "la capacidad de controlar y evaluar las capacidades de memoria actuales propias" (pág. 145) y como la capacidad que tiene el "cocimiento sobre el conocimiento". Por lo que es en la tercera infancia el espacio en el que se desarrollan actividades metacognitivas.

Para Flavell (2000i): es necesario entender que el metaconocimiento involucra los conocimientos y las experiencias metacognitivas que el niño interioriza sobre los contextos cognitivos en los que se desenvuelve, dichos conocimientos tienen más la característica de ser declarativos que 
procedurales, subdividiéndose en el conocimiento sobre las personas, tareas y estrategias:

El conocimiento sobre las personas involucra cualquier tipo de conocimiento que el niño puede interiorizar y estos difieren entre una y otra persona, el conocimiento de la tarea incluye la información que se dispone en el contexto en el que se encuentra el niño para que sea asimilada, esta información se genera de la experiencia metacognitiva, mientras que el conocimiento sobre las estrategias involucra la capacidad que tiene en nuestro caso el niño en la identificación de los procesos cognitivos que se involucran el proceso de resolución de problemas (Flavell, 2000j, pág. 147).

Así, se comprende que una estrategia cognitiva contribuye a la realización de la actividad cognitiva en cuestión, mientras que la estrategia metacognitiva es la que va ir generando la información sobre el trabajo que se va desarrollando o en sí mismo el progreso de la propia estrategia.

\subsection{Experiencia metacognitiva}

La experiencia metacognitiva se traduce como "el grueso del conocimiento metacognitivo, se refiere realmente a combinaciones 0 interacciones entre dos o tres de las categorías" (Flavell, 2000k, pág. 148), y por su naturaleza esta puede ser corta o larga, como también de carácter simple o complejo según el contenido que estas conllevan, estas experiencias cognitivas pueden producirse a lo largo de todo del proceso cognitivo que se emprende para la construcción de una tarea o la resolución de un problema.

\subsection{Resolución de problemas}

El resolver problemas en la vida diaria del ser humano es necesariamente cotidiano, en cualquier contexto que se encuentre, por ello es preciso que el niño pueda establecer un plan de resolución, en el que no necesariamente el objetivo principal sea la solución efectiva o no del problema, 
sino el proceso que el niño desarrolla dentro de aquel plan que estableció con la intención de solucionarlo, dicho esto de otra manera, que sea consciente de los diferentes pasos que realiza para poder resolver el problema, la consciencia que el niño posee de este proceso le permite volver sobre cada una de las fases del trabajo que ha desarrollado con el afán de poder corregirlo así se puede considerar que:

cualquier situación obstáculo que propicie la activación de un proceso cognitivo, que para ser resuelto necesita de la interacción de acciones que se puedan ejecutar, a partir de destrezas como: la observación, inferencia, suposición, análisis, etc., que emprende el ser humano como tal, permitiendo la adquisición de nuevas destrezas que posibilitan el desarrollo de un proceso que crea el camino para que los niños establezcan soluciones lógicas y reales al problema (Ullauri, 2013c, pág. 46).

A continuación, abordamos dos modelos de resolución de problemas el primero que propone Polya (1984a), y el segundo que define Sternberg (2011b). El modelo de Polya establece cuatro fases para la resolución de problemas matemáticos que en su orden son: i). entender el problema, ii). configurar un plan, iii). ejecutar el plan, iv). mirar hacia atrás (Polya, 1984b, pág. 51-53):

I. Entender el problema: esta comprensión del problema no implica solamente su lectura de forma literal, sus palabras, sus signos y símbolos, sino también lo implícito, lo que a primera vista no se mira, como las relaciones, categorías y variables que lo componen, ayudando al niño a poder comprender el problema en su totalidad. (Polya, 1984c; Ullauri, 2013d).

La génesis del proceso implica razonar sobre la tarea que se presenta para resolverla, reconociendo y entendiendo el problema en cuestión, esto implica que el niño debe partir del análisis crítico de las relaciones, categorías 
y variables para determinarlas en una primera fase, no será posible que el niño alcance esta fase si no ha podido establecer con suficiente profundidad las características del problema.

II. Un segundo momento es la configuración del plan, que incluye el mapeo de la situación problema que se requiere resolver, la estructuración de este plan requiere la puesta en escena de las destrezas y competencias pertinentes al tipo de problema que se intenta dar solución, en este caso el proceso metacognitivo de razonamiento hipotético como un medio que permita mirar en futuro hacia la construcción de una visualización sobre la resolución del problema:

que a modo de un reproductor de video puede ser repasada hacia adelante y hacia atrás, de forma que se podrán percibir los detalles más mínimos, que le permitirán decidir qué estrategia es la más adecuada para la resolución del problema, el volver hacia atrás y repasar de nuevo la escena y plantear hipótesis (Ullauri, 2013e, pág. 57).

Así se entiende que la naturaleza del razonamiento hipotético es metacognitiva.

III. Luego de la configuración del plan deviene la ejecución. La ejecución de un plan no garantiza la resolución del problema, lo que no se puede catalogar como un error o fracaso sino más bien como una oportunidad de replantear la estrategia, valorar errores e iniciar la planificación del nuevo plan y su ejecución.

La dinámica de estas fases como contenedoras de procesos cognitivos necesariamente involucra la consciencia de estos por parte del niño, lo que le faculta poder corregir errores sobre la situación. Durante la etapa de ejecución del plan, será capaz de detenerse sobre la marcha y replantear la estrategia 
que no está funcionando correctamente para poder continuar.

IV. Al final, mirar hacia atrás posibilita discriminar si la solución lograda es la solución correcta o no, este ejercicio que realiza el niño genera el espacio para poder volver a entender y comprender el planteamiento del problema, logrando la verificación que le satisfaga.

Polya (1984d): estima que el proceso de resolución de los problemas comprende dos tipos de razonamiento; el razonamiento regresivo y el razonamiento proyectivo, estos dos tipos de razonamiento se deben plasmar en la compresión del problema, la constitución del plan para resolverlo y sobre todo cuando no se genera la resolución del problema poder reformular las estrategias para tratar de resolverlo (pág. 134).

Desde esta perspectiva el autor considera que Polya:

buscamos de qué antecedentes se podría deducir el resultado deseado; después buscamos cual podría ser el antecedente de este antecedente, y así sucesivamente, hasta que, pasando de un antecedente a otro, encontremos finalmente alguna cosa conocida o admitida como cierta. Dicho proceso lo llamamos análisis, solución hacia atrás o razonamiento regresivo. En la síntesis, por el contrario, invirtiendo el proceso, partimos del último punto alcanzado en el análisis, del elemento ya conocido o admitido como cierto. Deducimos lo que en el análisis le precedía y seguimos así hasta que, volviendo sobre nuestros pasos, lleguemos finalmente a lo que se nos pedía. Dicho proceso lo llamamos síntesis, solución constructiva o razonamiento progresivo (Polya, 1984e, pág. 134).

El proceso resolutorio de problemas planteado por Sternberg (2011c): no es necesariamente orientado a la resolución de problemas matemáticos. Este proceso tiene un carácter cíclico que parte de: 1). identificación del problema, 2). definición y representación del mismo, 3). formulación de 
estrategias, 4). organización de la información, 5). ubicación de los recursos, 6). monitorización, 7). evaluación (pág. 430).

Figura 1. Ciclo de resolución del problema de Sternberg.

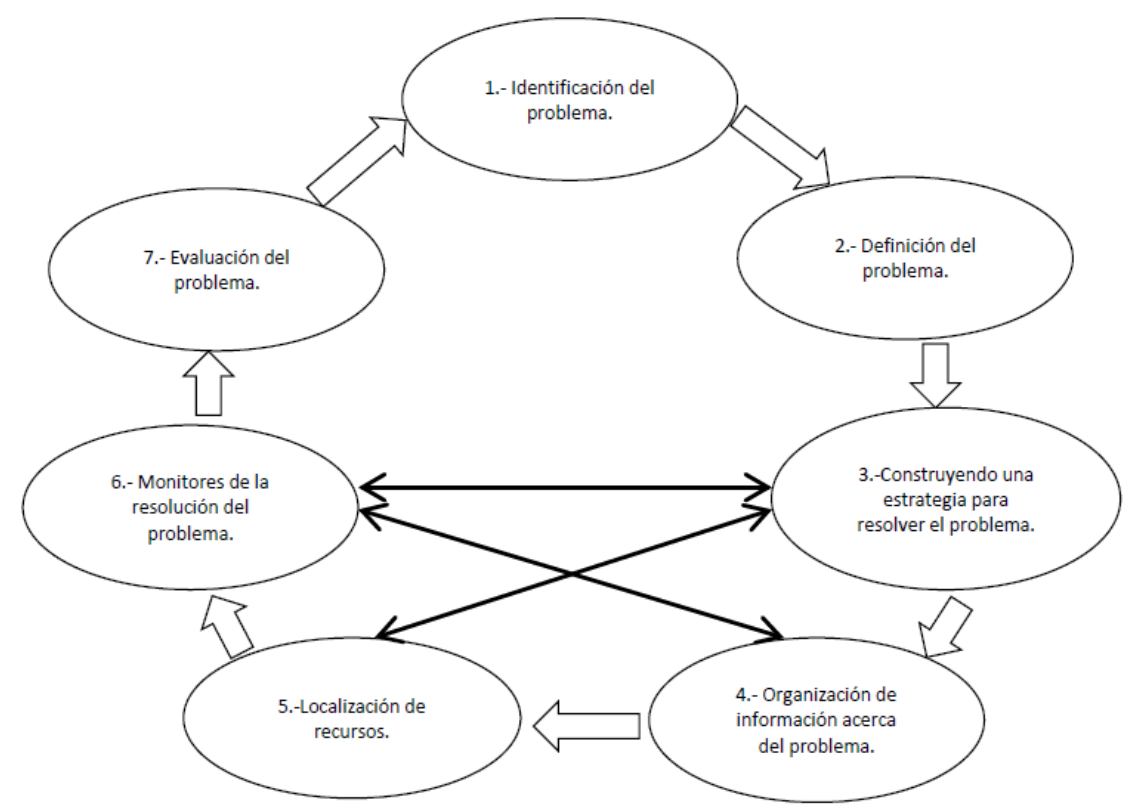
reversibilidad y los indicados por Polya (1984f): el razonamiento regresivo y proyectivo son correspondientes, identificándose estos como necesarios para 
poder resolver un problema con entera satisfacción el niño deberá haber desarrollado estos procesos básicos del pensamiento metacognitivo para comprender el problema, configurar el plan, establecer estrategias de resolución, desarrollar el plan para solucionarlo y en caso de no poder resolverlo, el razonamiento regresivo le posibilitará volver sobre el plan y las estrategias, para establecer mejoras o configurar un nuevo plan que le permita resolver el problema, en este sentido en un problema matemático se ven implicados todos los procesos cognitivos que hemos repasado, desde este punto el pensamiento lógico matemático juega un papel determinante.

\subsection{Pensamiento lógico matemático}

Para Castaño (2010): "el desarrollo del pensamiento lógico-matemático es el desarrollo de la capacidad de establecer relaciones y de operar con éstas" (pág. 96), comprendiéndose por esto que el desarrollo de este tipo de pensamiento comprende el involucramiento de procesos cognitivos que permitan el establecimiento de relaciones. En este sentido Piaget (1991c): sostiene que "el pensamiento del niño no se convierte en lógico más que por medio de la organización de sistemas de operaciones que obedecen a leyes de conjuntos comunes que son; composición, reversibilidad, la operación directa y su inversa, asociación de operaciones" (pág. 71), desde esta perspectiva se comprende al "pensamiento lógico matemático como el proceso cognitivo y metacognitivo generado por las interacciones de las experiencias y acciones en la solución de problema" (Ullauri, 2013f, pág. 46).

Según Saguillo (2008a): el pensamiento lógico matemático se fundamenta en la naturaleza y realidad objetiva que se expresa en proposiciones que adquieren un valor matemático pudiendo ser falsas 0 verdaderas, así "el pensamiento lógico matemático clásico se articula también

presuponiendo ciertas capacidades epistémicas de los seres humanos". (Saguillo, 2008b, pág. 6), que necesariamente son vinculantes con los 
procesos cognitivos y metacognitivos, permitiendo establecer relaciones de distinto orden ante las situaciones, objetos y conceptos, permitiendo que el niño pueda estructurar y restructurar la realidad, empleando procesos cognitivos y metacognitivos como el planteamiento de soluciones proyectivas a una determinada tarea, dicho de otra forma, el planteamiento de hipótesis.

La resolución de un problema de cualquier clase necesita el establecimiento de estrategias de resolución que se configuren en un plan, que necesariamente se construye sobre la base de procesos cognitivos y metacognitivos.

\section{Metodología}

El presente trabajo contempló el estudio bibliográfico pertinente sobre el desarrollo del razonamiento lógico en la tercera infancia y como este proceso metacognitivo se ve inmerso en la resolución de problemas. La indagación bibliográfica se realizó entre los meses de septiembre y octubre del 2017, para ello se han empleado algunos descriptores que comprenden: desarrollo cognitivo en la segunda y tercera infancia, metacognición y procesos metacognitivos, razonamiento hipotético, pensamiento lógico matemático y resolución de problemas. De las fuentes consultadas se seleccionaron aquellas que informen sobre los aspectos base que evidencian el desarrollo cognitivo y metacognitivo de los niños y de manera espacial los que implícitamente se ven relacionados con la resolución de problemas matemáticos.

\section{Conclusiones}

La realización de este trabajo ha permitido llegar a algunas conclusiones claves en el proceso de desarrollo del razonamiento hipotético como un proceso metacognitivo para la resolución de problemas, estas conclusiones se concretizan en: 
- Los procesos metacognitivos son producto de la suma de los procesos cognitivos, así el surgimiento del razonamiento hipotético se ve inherentemente unido a la suma de destrezas cognitivas básicas como la percepción, atención, memoria, pensamiento, razonamiento y lenguaje.

- Se evidencia que el tratamiento de las tareas a las que se enfrentan los niños son consideradas por ellos como problemas, que no necesariamente son conocidas por estos y que muy probablemente las podrán resolver por medio del desarrollo de algunas destrezas cognitivas como: identificación del problema, capacidad de definición y representación, formulación de estrategias, organización de la información, ubicación de los recursos, monitorización el proceso, establecimiento inferencias, evaluación y de forma especial por el desarrollo de la destreza metacognitiva de razonamiento hipotético como un proceso del pensamiento lógico matemático.

- Se pone en contraste los estudios teóricos de Piaget (1991d), Flavell (2000m), Sternberg (2011e) y Hacker (1988b) en relación con la perspectiva de resolución de problemas de Polya (1984g): pudiéndose constatar que la función que conllevan los procesos cognitivos y metacognitivos como el razonamiento hipotético permite configurar un plan y sus estrategias de manera proyectiva para la resolución de problemas de diferente índole.

\section{Referencias}

Bruner, J. (1995). Escuelas para pensar. Madrid: Paidós.

Castaño, J. (2010). La matemática en Transición y Primer Grado de Escuela Nueva. Manual de implementación escuela nueva, generalidades y Orientaciones Pedagógicas para Transición y Primer 
Grado. Tomo I. Bogotá, Colombia: Ministerio de Educación Nacional. ISBN: 978-958-8712-41-3, págs. 212. Recuperado de: https://www.mineducacion.gov.co/1759/articles340089 archivopdf orientaciones pedagogicas tomol.pdf Dorsch, F. (2005). Diccionario de Psicología. Barcelona: Heder. Flavell, J. (2000a,b,c,d,e,f,g,h,i,j,k,l,m). El Desarrollo cognitivo. Madrid: Visor.

Hacker, D. (1998a,b). Metacognition: Definitions and empirical foundations. En Metacognition in educational theory and practice. EE. UU.: The University of Memphis, pp. 1-23. Recuperado de: http://vcell.ndsu.nodak.edu/ ganesh/seminar/Hacker Metacognition\% 20-\%20Definitions\%20and\%20Empirical\%20Foundations.htm

Piaget, J. (1991a,b,c,d). Seis estudios de Piscología. Barcelona: Labor. Polya, G. (1984a,b,c,d,e,f,g). Cómo plantear y resolver problemas. México: Trillas.

Saguillo, J. (2008a,b). El pensamiento lógico-matemático. Madrid: Akal. Sternberg, R. (2011a,b,c,d,e). Psicología cognitiva. México: Thomson. Ullauri, J. (2013a,b,c,d,e,f). Proceso metacognitivo del pensamiento lógico matemático: razonamiento hipotético. Cuenca, Ecuador: Universidad de Cuenca, págs. 207. Recuperado de:

https://www.google.com/url?sa=t\&rct=j\&q=\&esrc=s\&source=web\&cd=1 \&cad=rja\&uact=8\&ved=0ahUKEwipgOLRj6fPAhUGkh4KHakqA5oQFg gdMAA\&url=http://dspace.ucuenca.edu.ec/bitstream/123456789/20783 11/Tesis.pdf\&usg=AFQjCNHRImzZhF8Tr5MmWWUQv4TNz8O-0Q Wellman, H. (1985). The origins of metacognition. In D.L. Forrest-Pressley, G.E. MacKinnon, \& T.G. Waller (Eds.), Metacognition, cognition and human performance, pp. 1-31. Orlando, FL: Academic Press. 
Jaime Iván Ullauri Ullauri

e-mail: jaime.ullauri@unae.edu.ec

Nacido en Cuenca, Ecuador. Lcdo. en Psicología

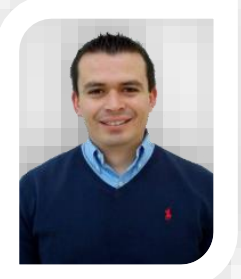

Educativa, Magister en Educación y Desarrollo del Pensamiento (Universidad de Cuenca-Ecuador), Magister en Orientación Educativa (UNED-España) y Doctorante del programa de Psicología de la Educación de la Universitat de Barcelona. Docente-Investigador en la cátedra de Aprendizaje Humano y de Nivelación General en Universidad Nacional de Educación, ha participado en procesos similares en la Universidad de Cuenca. Docente de Educación General Básica y Bachillerato en Estudios Sociales y Desarrollo del Pensamiento Filosófico. Facilitador Académico dentro de los programas de Asesoría y Auditoría Educativa y Formación Continua "Sí Profe" del Ministerio de Educación del Ecuador. 
Carol Ivone Ullauri Ullauri

e-mail: carol.ullauri@unae.edu.ec

Nacida en Cuenca, Ecuador. Lic. en Psicología Educativa Especialización Orientación Profesional, : đ: Magister en Psicología de la Educación y Desarrollo Humano en contextos multiculturales (Universidad de Valencia-España). Docente-Investigador en la cátedra de Neurociencias en Universidad Nacional de Educación. Docente-Investigador en las cátedras de Teorías del Aprendizaje, Psicología del Desarrollo Humano, Teorías y Sistemas Psicológicos y Psicología General en la Universidad de Cuenca. Docente de Nivelación de saberes en la Universidad de Cuenca. Docente de Educación General Básica y Bachillerato en Lengua y Literatura.

El contenido de este manuscrito se difunde bajo una Licencia de Creative Commons ReconocimientoNoComercial-Compartirlgual 4.0 Internacional 